\title{
Multi-Frame Image Processing of Himawari-8/AHI Data
}

\author{
By Toshiro SugimurA, ${ }^{1)}$ Yuuki UChIDA, ${ }^{1)}$ Sadayoshi Aoyama, ${ }^{1)}$ Tomohito AsAKA, ${ }^{1)}$ and Keishi IwASHITA ${ }^{1)}$ \\ ${ }^{1)}$ College of Industrial Technology, Nihon University, Chiba, Japan
}

(Received June 22nd, 2017)

\begin{abstract}
The Japan Meteorological Agency successfully launched Himawari-8 in October 2014, and began operations in July 2015. A new sensor system, Advanced Himawari Imager (AHI), is observing the surface of the Japan area frequently (every $2.5 \mathrm{~min}$ ) and 24 images can be observed sequentially each hour. This study examined the feasibility of multi-frame image processing using Himawari-8/AHI data obtained over one hour. Slight distortion of images was adjusted by dividing one pixel into ten subpixels and by applying automatic registration, which facilitated multi-frame image processing. As a result, Himawari-8/AHI data is improved effectively to allow closer observation of the details of an image.
\end{abstract}

Key Words: Himawari-8/AHI, Sequential Observation, Automatic Registration, Multi-Frame Processing

\section{Introduction}

Himawari-8 is Japan's next-generation geostationary meteorological satellite using a highly improved sensor, Advanced Himawari Imager (AHI). The satellite observes all of the Earth's surface (within visual range) every 10 min and all of Japan every $2.5 \mathrm{~min}$. Therefore, 24 images of Japan can be observed sequentially in an hour.

Tasks performed by Himawari-8, operated by Japan Meteorological Agency (JMA), include weather monitoring by collecting information about cloud coverage and movement. Clouds appear, move, and dissipate slowly, and this process can be observed using Himawari-8/AHI sequential images. On the other hand, because many objects on the ground are fixed, Hiamawari-8 also provides multi-frame images of immovable objects on the ground.

The authors apply high-resolution image reconstruction using multi-frame low resolution images in this paper. This technique was developed to enhance video images. Since a set of Himawari-8/AHI sequential data is considered to be a multi-frame image, high-resolution reconstruction processing was examined as an enhancement opinion.

Comparing 24 images observed sequentially in an hour does not yield noticeable distortion among them. If the pixels in each images are divided into $10 \times 10$ subpixels, several distortions, particularly in Band 3, become apparent because the resolution in Band 3 is higher than that of other bands. If the distortion of images divided using subpixels corrected automatically, subpixels can be fixed in their positions relative to each other to produce an enhanced image.

This study examined the feasibility of multi-frame image processing using Himawari-8/AHI data observed sequentially over an hour. By adjusting the distortion of multi-frame images by subpixel comparison and automatic registration, it is possible to improve image quality from multi-frame image processing using mean value, median value, or a regression function to shift subpixel positions in an image. As a result,
Himawari-8 data can be improved to observe more information than provided by the raw data.

\section{Test Data and Test Site}

The JMA successfully launched Himawari-8 on October 7, 2014 and the satellite began operations on July 7, 2015, replacing the previous Himawari-7 satellite. The JMA is operating Himawari-8 at approximately $140^{\circ}$ east, covering the East Asia and Western Pacific regions. A new sensor system called AHI is scanning five areas: Full Disk (images of the whole Earth as seen from the satellite), the Japan Area, the Target Area, and two Landmark Areas. In each 10-min period, the AHI is able to scan not only the Full Disk once but also the Japan Area four times. The AHI is observing the surface of Japan every 2.5 min sequentially. Additionally, the number of bands has increased from 5 (VIS 1, IR 4) to 16 (VIS 3, NIR

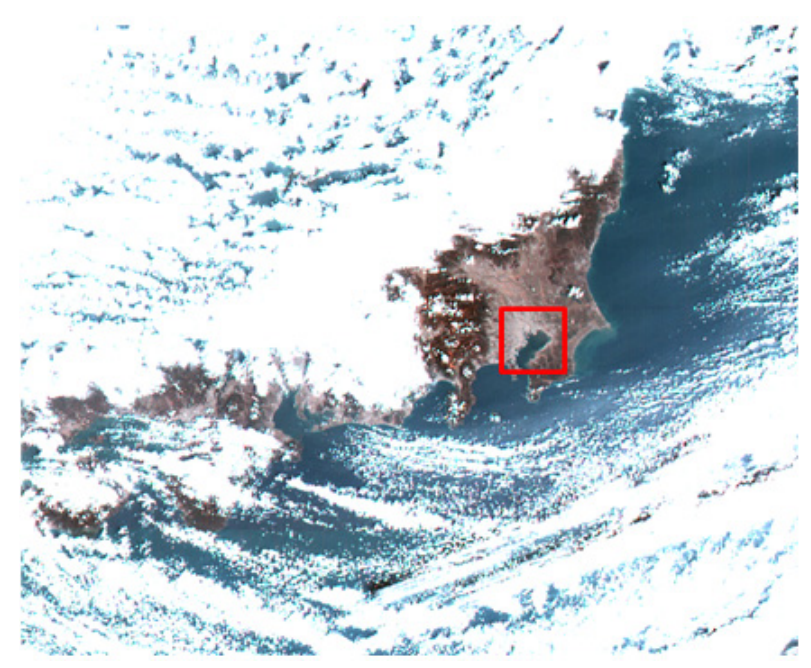

Fig. 1. Japan area observed by Himawari-8/AHI on December 4, 2015 (09:00 JST). A composite image is produced by assigning red to Band 3, green to Band 2 and blue to Band 1. The red box in this figure shows the test site of this study. 
3 and IR 10).

The Himawari-8/AHI data observed on December 4, 2015 from 9 to 10 a.m. (JST) are used in this study. The weather was clear in the Kanto area at that time (Fig. 1). Large clouds rarely appeared, but small clouds appeared over short periods of time.

\section{Procedure}

\subsection{Registration}

Image-to-image registration was conducted for the images observed in an hour. First, one pixel was divided into ten subpixels, and an image composed of subpixels was created. Two resampling methods, nearest neighbor and cubic convolution, were applied to determine the corresponding point that indicates a difference between images. Second, a correlation coefficient was calculated after shifting the slave image by one subpixel unit, and the corresponding point was investigated. As a result, the correlation coefficient was the highest at the center point (not the shifting position) in any combination and regardless of the resampling method applied. However, when the corresponding point was considered as a peak point approximated by a quadratic polynomial, maximum distortions of 0.3 and 0.5 pixels were detected for Bands 1,2, and 4, and for Band 3, respectively. Table 1 shows the distortions detected using a quadratic polynomial function, and Figure 2 shows the distortion of corresponding points for subpixel shifting direction-for pixels (toward east) and lines (toward south). In general, the distortion of Band 3 is twice that of Bands 1,2, and 4. This is because the spatial resolution of Band $3(500 \mathrm{~m})$ is higher than that of Bands 1, 2, and $4(1 \mathrm{~km})$.

Table 1. Distortions measured using the peak of the regressed quadratic polynomial of correction coefficients.

\begin{tabular}{|c|c|c|c|c|c|}
\hline \multirow{2}{*}{ No. } & \multirow{2}{*}{2015.12 .4} & \multicolumn{2}{|c|}{ Band2 } & \multicolumn{2}{|c|}{ Band3 } \\
\hline & & Pixel & Line & Pixel & Line \\
\hline 1 & 9:00 & 0.00 & 0.00 & 0.00 & 0.00 \\
\hline 2 & 9:03 & 0.13 & -0.66 & -0.09 & -1.24 \\
\hline 3 & $9: 05$ & -0.34 & -0.81 & -0.99 & -1.47 \\
\hline 4 & 9:08 & -0.13 & -1.79 & -0.06 & -3.79 \\
\hline 5 & $9: 10$ & -1.92 & 0.21 & -4.37 & 0.65 \\
\hline 6 & $9: 13$ & -1.85 & -0.58 & -3.72 & -0.91 \\
\hline 7 & $9: 15$ & -1.56 & -1.39 & -3.22 & -2.53 \\
\hline 8 & $9: 18$ & -1.36 & -2.25 & -3.22 & -5.32 \\
\hline 9 & $9: 20$ & -0.97 & -0.82 & -2.29 & -1.34 \\
\hline 10 & $9: 23$ & -0.73 & -1.18 & -1.87 & -2.20 \\
\hline 11 & $9: 25$ & -0.40 & -2.00 & -1.52 & -4.15 \\
\hline 12 & $9: 28$ & -0.78 & -2.31 & -2.09 & -5.10 \\
\hline 13 & $9: 30$ & -1.03 & -0.14 & -1.98 & 0.01 \\
\hline 14 & $9: 33$ & -0.53 & -0.45 & -1.24 & -0.52 \\
\hline 15 & $9: 35$ & -1.00 & -1.27 & -2.38 & -1.89 \\
\hline 16 & $9: 38$ & -0.48 & -1.58 & -1.38 & -2.86 \\
\hline 17 & $9: 40$ & 0.84 & -0.01 & 1.61 & 0.42 \\
\hline 18 & $9: 43$ & 1.48 & -0.95 & 3.42 & -1.22 \\
\hline 19 & $9: 45$ & 1.41 & -1.48 & 2.54 & -2.39 \\
\hline 20 & $9: 48$ & 1.36 & -1.86 & 3.25 & -3.62 \\
\hline 21 & $9: 50$ & 0.87 & 0.37 & 1.97 & 1.44 \\
\hline 22 & $9: 53$ & 1.03 & -0.57 & 1.85 & -0.14 \\
\hline 23 & $9: 55$ & 0.47 & -0.92 & 0.83 & -0.88 \\
\hline 24 & $9: 58$ & 0.46 & -1.38 & 0.85 & -1.71 \\
\hline
\end{tabular}

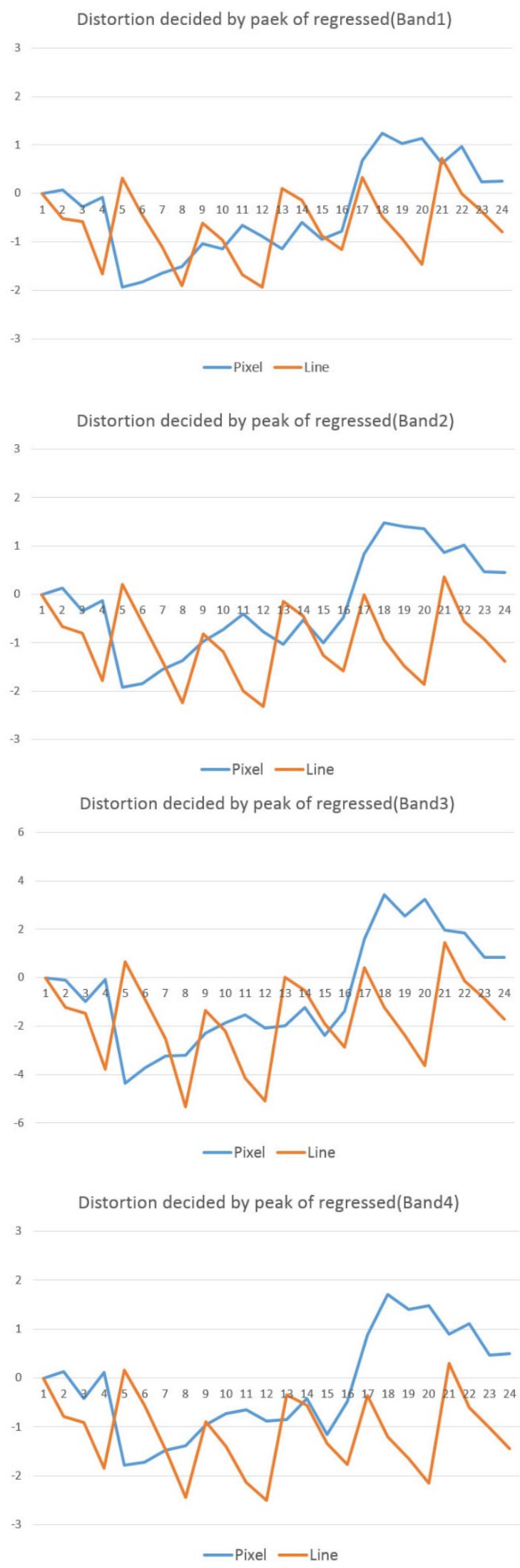

Fig. 2. Distortion measured using the peak of a quadratic polynomial approximation of correlation coefficients. The upper figure is a result for Band 1 and the lower figure is Band 4. Horizontal axis shows time from 9 to $10 \mathrm{a} . \mathrm{m}$. and the vertical axis shows distortions by subpixel unit. 


\subsection{Correction of brightness}

There are differences in the brightness levels and standard deviation of the 24 images taken over one hour, which are caused by changes in the sun elevation. To process multi-frame images, it is best to normalize the brightness of each image so images are approximately equally bright. Changes in DN (brightness) are observed as mean values at each observation time, as shown in Table 2 . The mean values increase from 146 to 175 and from 105 to 130 in Band 2 and Band 3, respectively. Standard deviations also increase from 15 to 23 and from 24 to 35 in Band 2 and Band 3, respectively. The average value and standard deviation of the brightness were adjusted using a simple pre-processing formula as follows.

$$
\mathbf{D N}_{\text {ner }}=\frac{\sigma_{9: 30}}{\sigma}(\mathbf{D N}-\overline{\mathbf{D N}})+\overline{\mathbf{D N}}_{9: 30},
$$

where $\mathrm{DN}$ and $\mathrm{DN}_{\text {new }}$ are Digital Number of image, $\overline{\mathrm{DN}}$ and $\sigma$ are mean and standard deviation and $\overline{\mathrm{DN}}_{9: 30}$ and $\sigma_{9: 30}$ are mean and standard deviation at 9:30.

The influence of the sun elevation can be expressed as the cosine of the solar zenith angle. Table 3 shows the sun elevation and cosine value of the zenith angle for each observation time. The correlation of DN mean values and the cosine of the zenith angle was 0.999 . The simple method corrects the influence of the zenith angle so that brightness of the images observed in an hour is similar.

Table 2. Mean and standard deviation of the DN values observed in an hour.

\begin{tabular}{|c|c|c|c|c|c|}
\hline \multirow{2}{*}{ No. } & \multirow{2}{*}{2015.12 .4} & \multicolumn{2}{|c|}{ B02 } & \multicolumn{2}{|c|}{ B03 } \\
\hline & & mean & st.dev. & mean & st.dev. \\
\hline 1 & 9:00 & 145.91 & 15.49 & 105.64 & 24.50 \\
\hline 2 & 9:03 & 147.28 & 15.85 & 106.74 & 24.98 \\
\hline 3 & 9:05 & 148.65 & 16.20 & 107.89 & 25.52 \\
\hline 4 & 9:08 & 149.95 & 16.60 & 108.92 & 26.04 \\
\hline 5 & $9: 10$ & 151.37 & 16.89 & 110.26 & 26.51 \\
\hline 6 & $9: 13$ & 152.67 & 17.30 & 111.44 & 27.12 \\
\hline 7 & $9: 15$ & 153.94 & 17.60 & 112.43 & 27.61 \\
\hline 8 & $9: 18$ & 155.20 & 17.94 & 113.48 & 28.06 \\
\hline 9 & $9: 20$ & 156.63 & 18.24 & 114.81 & 28.61 \\
\hline 10 & $9: 23$ & 157.99 & 18.61 & 115.88 & 29.13 \\
\hline 11 & $9: 25$ & 159.16 & 18.92 & 116.85 & 29.62 \\
\hline 12 & $9: 28$ & 160.35 & 19.21 & 117.88 & 30.04 \\
\hline 13 & $9: 30$ & 161.85 & 19.52 & 119.36 & 30.55 \\
\hline 14 & $9: 33$ & 163.12 & 19.90 & 120.38 & 31.06 \\
\hline 15 & $9: 35$ & 164.28 & 20.28 & 121.36 & 31.65 \\
\hline 16 & $9: 38$ & 165.53 & 20.56 & 122.45 & 32.05 \\
\hline 17 & $9: 40$ & 166.81 & 20.87 & 123.66 & 32.51 \\
\hline 18 & $9: 43$ & 167.99 & 21.15 & 124.63 & 32.83 \\
\hline 19 & $9: 45$ & 169.12 & 21.48 & 125.53 & 33.31 \\
\hline 20 & $9: 48$ & 170.07 & 21.79 & 126.50 & 33.80 \\
\hline 21 & $9: 50$ & 171.55 & 21.97 & 127.83 & 34.25 \\
\hline 22 & $9: 53$ & 172.52 & 22.35 & 128.67 & 34.73 \\
\hline 23 & $9: 55$ & 173.58 & 22.67 & 129.54 & 35.21 \\
\hline 24 & $9: 58$ & 174.53 & 23.03 & 130.48 & 35.71 \\
\hline
\end{tabular}

Table 3. Sun elevation and cosine of zenith angle observed in an hour.

\begin{tabular}{|c|c|c|c|c|}
\hline \multirow{2}{*}{ No. } & \multirow{2}{*}{2015.12 .4} & \multicolumn{3}{|c|}{ Sun } \\
\hline & & Elevation & Azimuth & $\cos$ (zenith A.) \\
\hline 1 & 9:00 & 21.84 & 142.19 & 0.3720 \\
\hline 2 & 9:03 & 22.15 & 142.71 & 0.3770 \\
\hline 3 & $9: 05$ & 22.46 & 143.24 & 0.3820 \\
\hline 4 & 9:08 & 22.76 & 143.78 & 0.3869 \\
\hline 5 & $9: 10$ & 23.06 & 144.32 & 0.3917 \\
\hline 6 & $9: 13$ & 23.35 & 144.86 & 0.3963 \\
\hline 7 & $9: 15$ & 23.64 & 145.40 & 0.4010 \\
\hline 8 & $9: 18$ & 23.93 & 145.95 & 0.4056 \\
\hline 9 & $9: 20$ & 24.21 & 146.50 & 0.4101 \\
\hline 10 & $9: 23$ & 24.49 & 147.06 & 0.4145 \\
\hline 11 & $9: 25$ & 24.76 & 147.62 & 0.4188 \\
\hline 12 & $9: 28$ & 25.03 & 148.18 & 0.4231 \\
\hline 13 & $9: 30$ & 25.30 & 148.75 & 0.4274 \\
\hline 14 & $9: 33$ & 25.56 & 149.32 & 0.4315 \\
\hline 15 & $9: 35$ & 25.82 & 149.90 & 0.4355 \\
\hline 16 & $9: 38$ & 26.07 & 150.48 & 0.4395 \\
\hline 17 & $9: 40$ & 26.32 & 151.06 & 0.4434 \\
\hline 18 & $9: 43$ & 26.56 & 151.65 & 0.4471 \\
\hline 19 & $9: 45$ & 26.80 & 152.24 & 0.4509 \\
\hline 20 & $9: 48$ & 27.03 & 152.83 & 0.4545 \\
\hline 21 & $9: 50$ & 27.26 & 153.42 & 0.4580 \\
\hline 22 & $9: 53$ & 27.49 & 154.02 & 0.4616 \\
\hline 23 & $9: 55$ & 27.71 & 154.63 & 0.4650 \\
\hline 24 & $9: 58$ & 27.92 & 155.24 & 0.4682 \\
\hline
\end{tabular}

\subsection{Multi-frame processing}

Multi-frame image processing is a technique to construct high-resolution images from multiple low-resolution images having different subpixel shifts. Correction by multi-frame processing was carried out using images observed over an hour that were adjusted for position and brightness. In this study, the authors attempted multi-frame processing based on mean value or intermediate value replacement. These corrections were processed based on a subpixel unit because adjacent subpixels will be affected to a greater extent and will produce better combinations than original pixel values. Ideally, the multi-frame processing image will become a high-resolution, smooth image. Figures 3 and 4 show the results of the processing method used for Band 2 and Band 3, respectively. Figures 3(a), 3(d), 4(a), and 4(d) are the images observed at 9:00 a.m., Figs. 3(b), 3(e), 4(b), and 4(e) are the images observed at 9:30 a.m., Figs. 3(c), 3(f), 4(c), and 4(f) are the images observed at 9:58, and Figs. $3(\mathrm{~g}), 3(\mathrm{~h}), 4(\mathrm{~g})$, and 4(h) are the images produced by multi-frame image processing. Improvement of image quality by exhibiting finer resolution and enhancement effects such as removal of moving small clouds appearing in a short time can be observed in Figs. 4(g) and 4(h). However, although a slight improvement in resolution can be observed, it was not possible to obtain a major improvement.

Point "A" shown in Figs. 4(a), 4(b), 4(d), and 4(e) might be a cloud moving and disappearing. There are also noisy patterns in Tokyo Bay, which are reduced and smoothed in the reconstructed images in Fig. 3(g) and particularly Fig. 3(h). 
Trans. JSASS Aerospace Tech. Japan Vol. 16, No. 3 (2018)

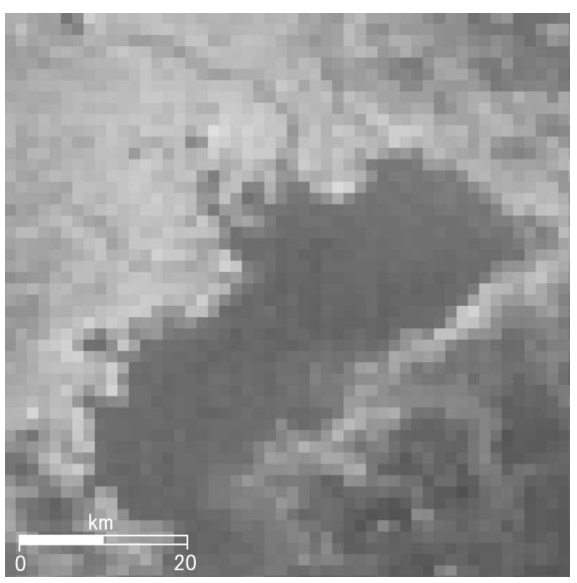

3(a) 2015.12.4 0000Z (NN)

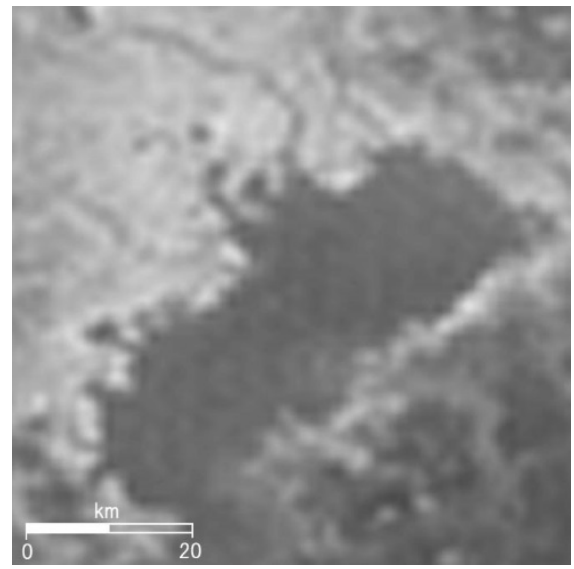

3(d) 2015.12.4 0000Z (CC)

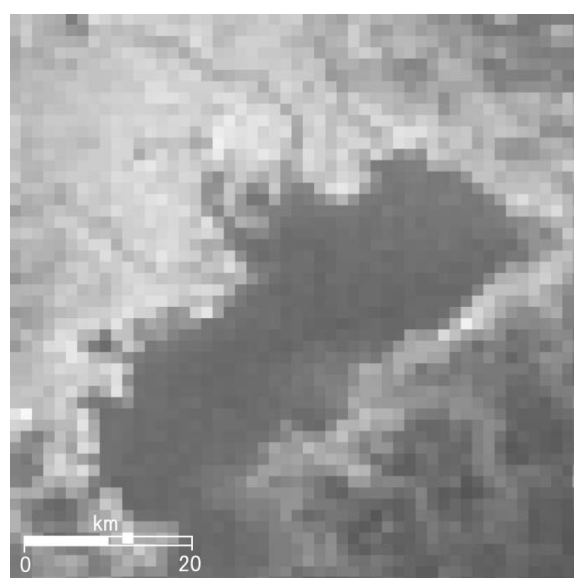

3 (b) 2015.12.4 0030Z (NN)

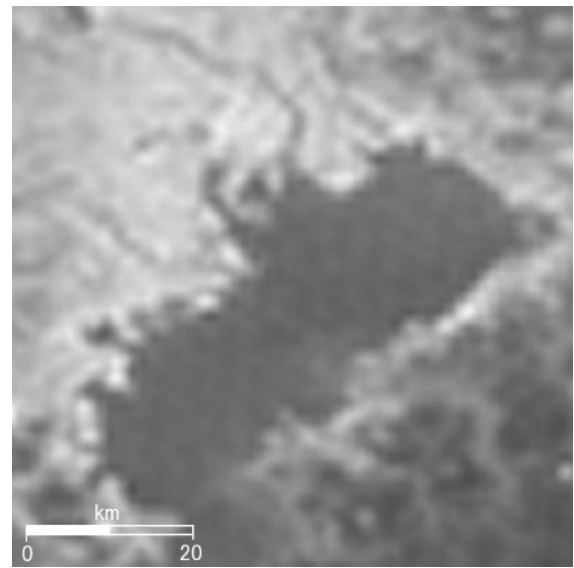

3(e) 2015.12.4 0030Z (CC)

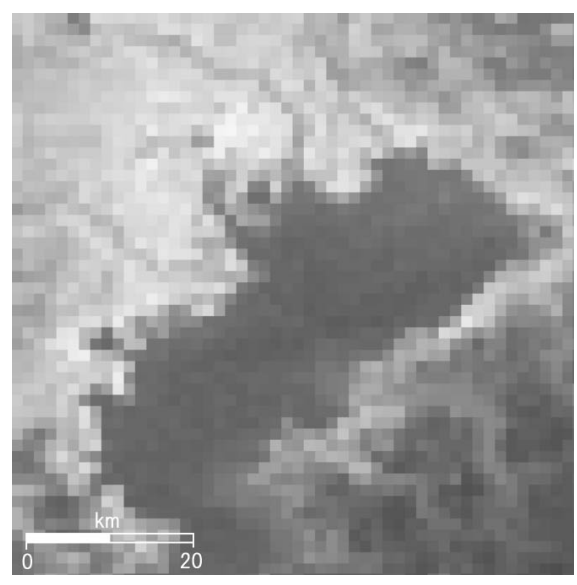

3(c) 2015.12.4 0058Z (NN)

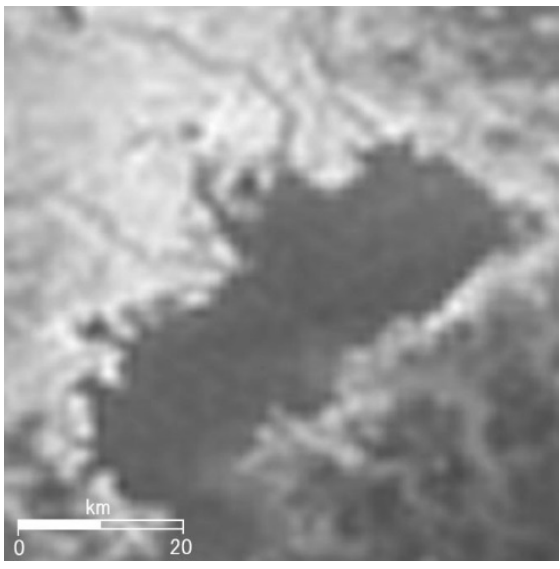

3(f) 2015.12.4 0058Z (CC)

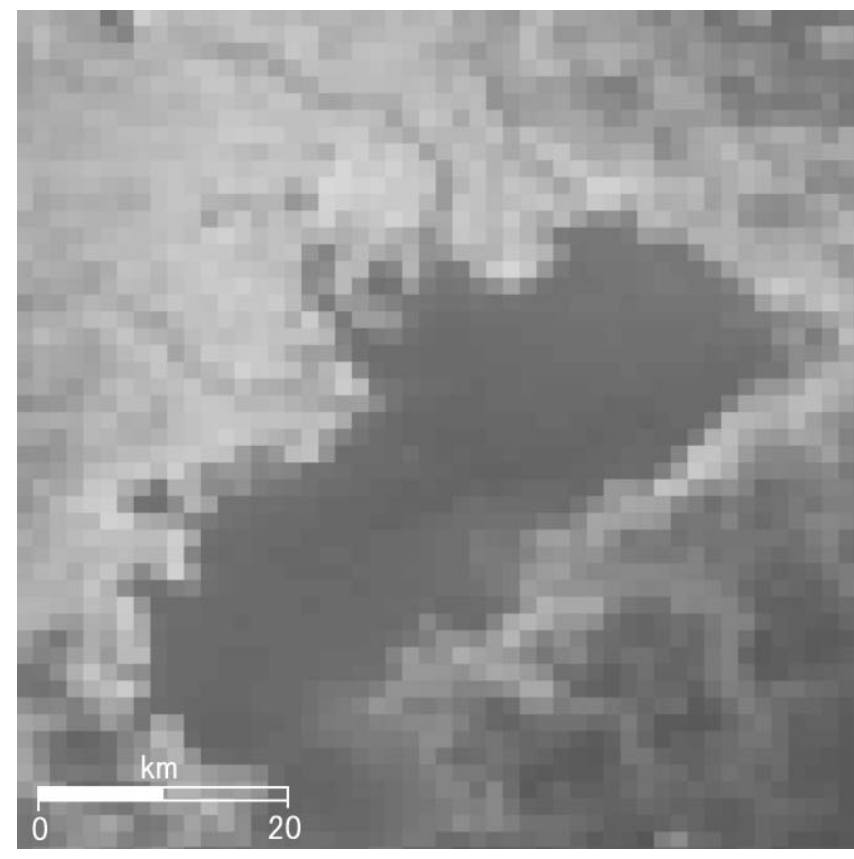

3(g) 2015.12.4 0000-0058Z processed (NN)

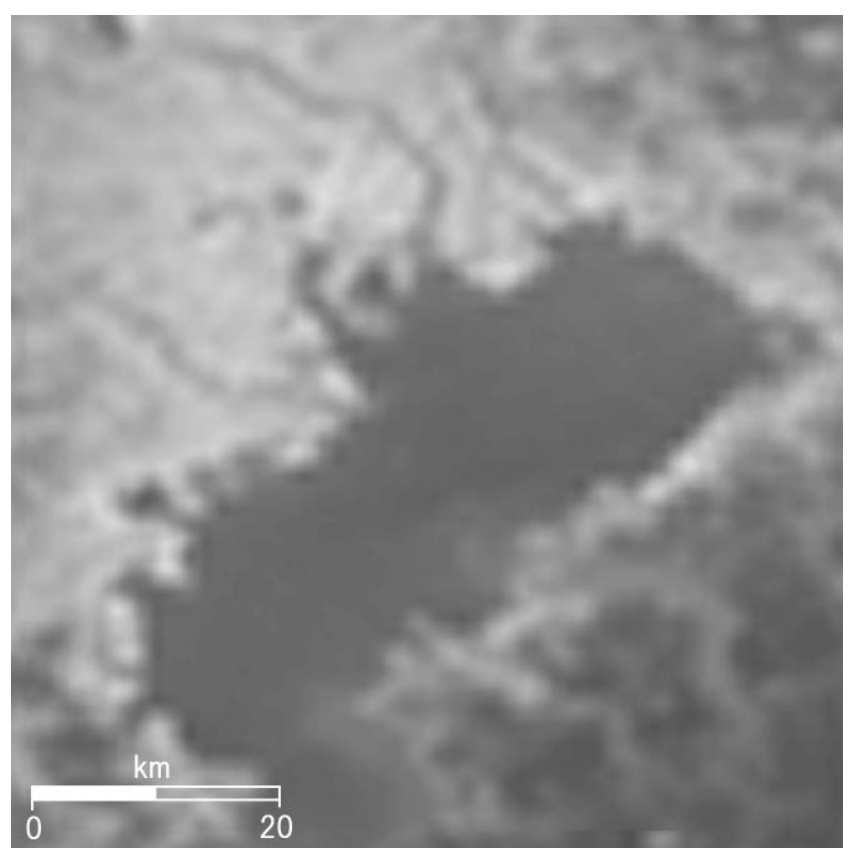

3(h) 2015.12.4 0000-0058Z processed (CC)

Fig. 3. Results of multi-frame processing for Band 2; images observed on Dec. 4, 2015. Upper images are images observed at 0000Z(a, d), 0030Z(b, e), and $0058 \mathrm{Z}(\mathrm{c}, \mathrm{f})$ by two different sampling methods (NN: nearest neighbor, $\mathrm{CC}$ : cubic convolution). Lower images are multi-frame processing results using images observed in an hour. 
Trans. JSASS Aerospace Tech. Japan Vol. 16, No. 3 (2018)

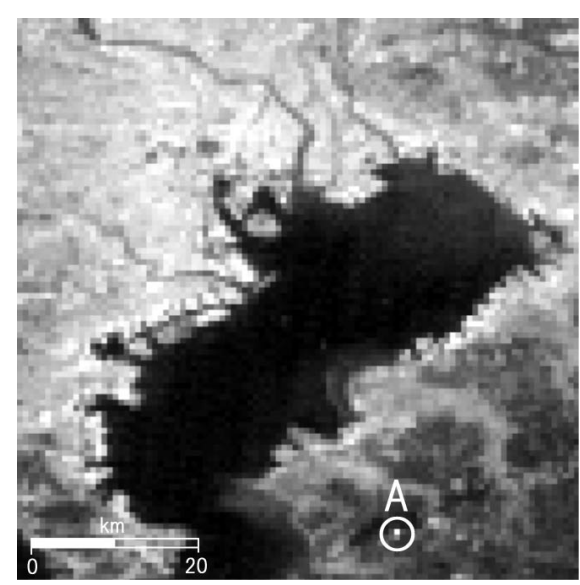

4(a) 2015.12.4 0000Z (NN)

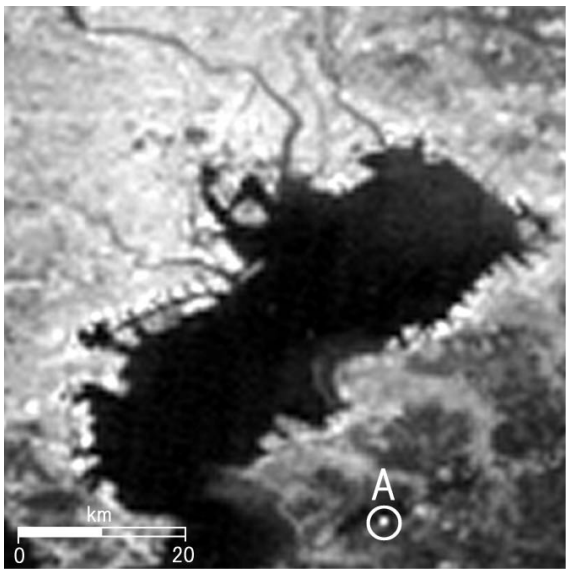

4(d) 2015.12.4 0000Z (CC)

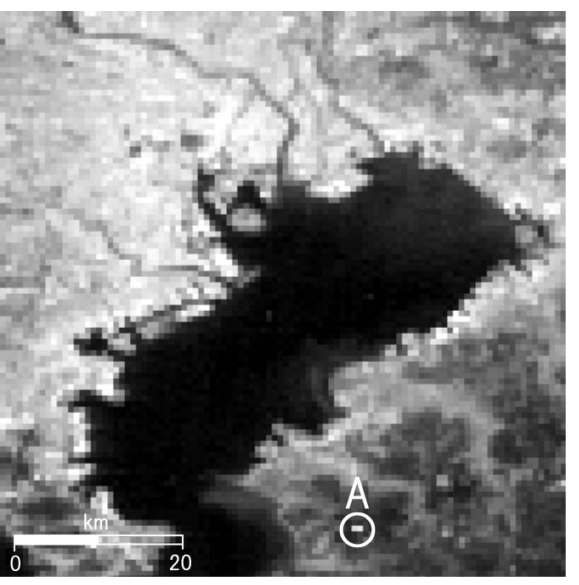

4(b) 2015.12.4 0030Z (NN)

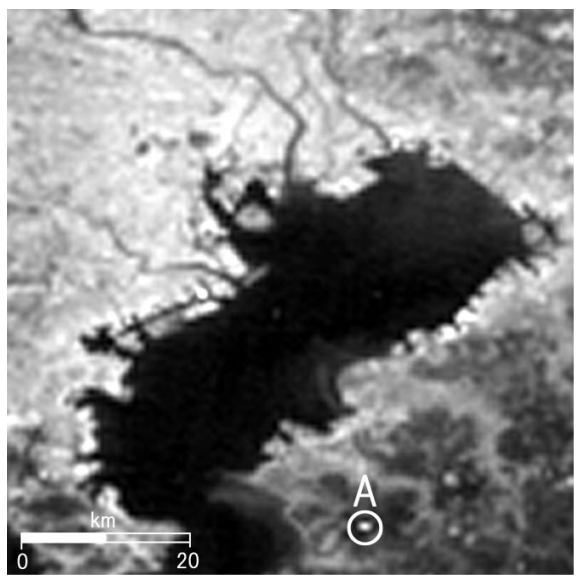

4(e) 2015.12.4 0030Z (CC)

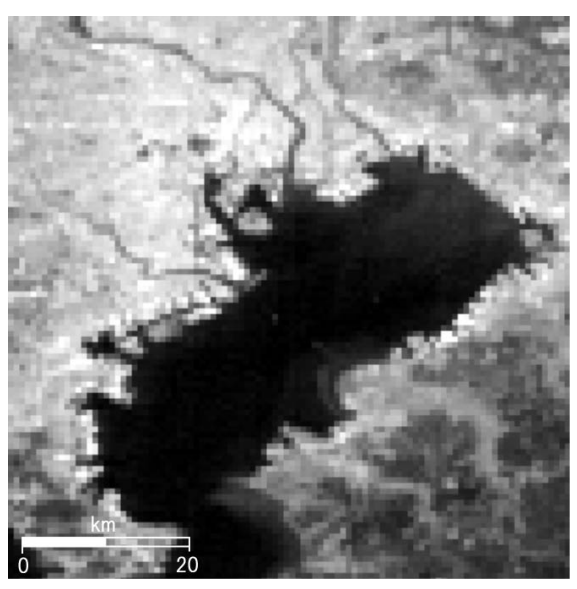

4(c) 2015.12.4 0058Z (NN)

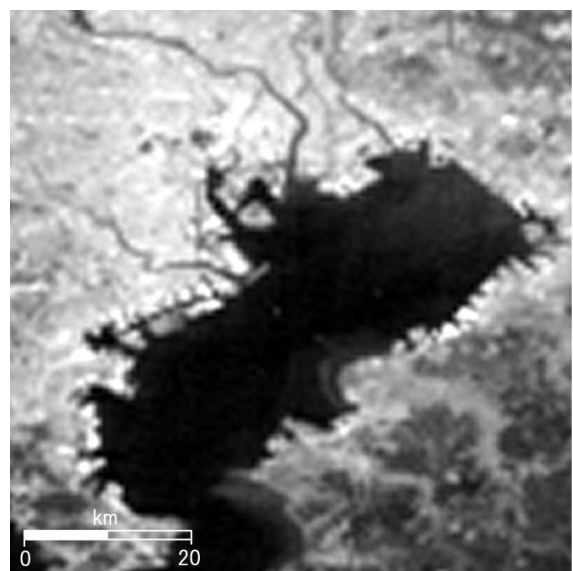

4(f) 2015.12.4 0058Z (CC)

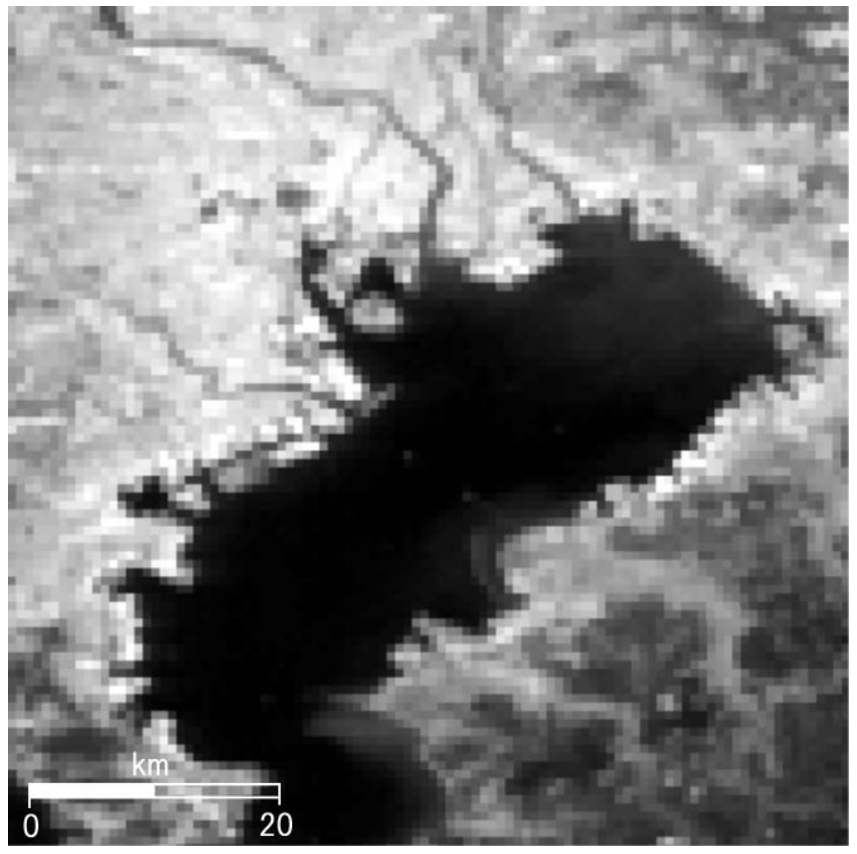

4(g) 2015.12.4 0000-0058Z processed (NN)

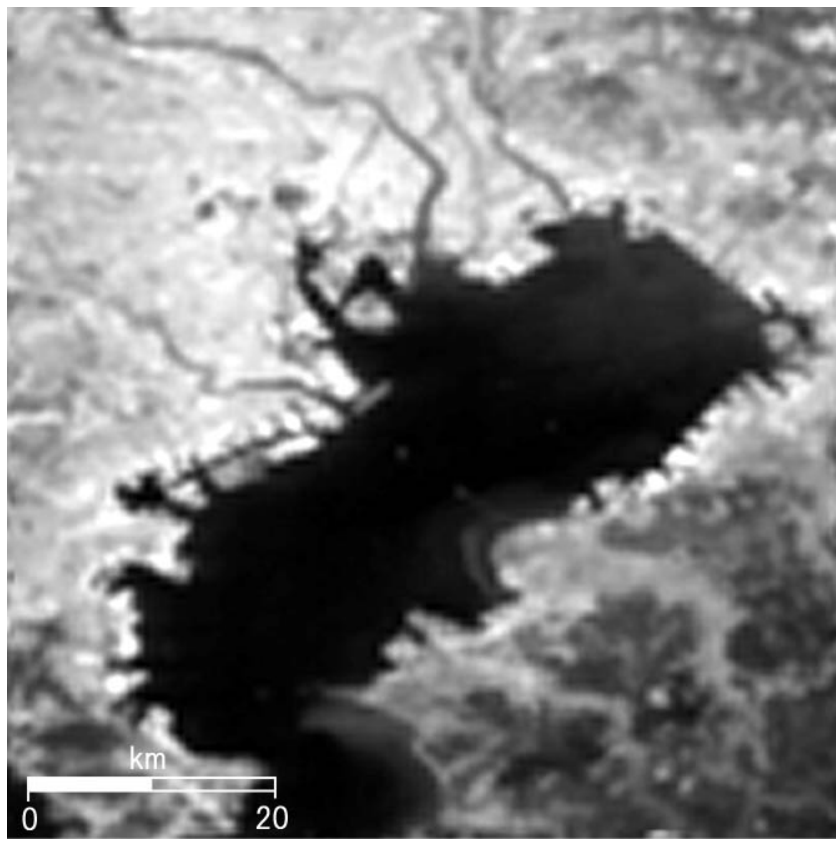

4(h) 2015.12.4 0000-0058Z processed (CC)

Fig. 4. Results of multi-frame processing for Band 3; images observed on Dec. 4, 2015. Upper images are images observed at 0000Z(a, d), 0030Z(b, e), and $0058 \mathrm{Z}(\mathrm{c}, \mathrm{f})$ by two different sampling methods (NN: nearest neighbor, CC: cubic convolution). Lower images are multi-frame processing results using images observed in an hour. 


\section{Results and Discussion}

The following results are summarized.

(1) Image-to-image registration is possible based on pixels divided into $10 \times 10$ subpixels.

(2) In the resampling method for subpixel image generation, neither the nearest neighbor method nor cubic convolution method had any effect when searching for corresponding points.

(3) During image-to-image registration, distortion levels were similar on each band.

(4) Improvement in image quality was confirmed by confirming finer resolution in the reconstructed images.

(5) It was possible to remove small clouds appearing for a short time.

The differences in the resampling methods did not have a major influence on searching for the corresponding point. The maximum distortion was 3 subpixels for Bands 1, 2, and 4, and 6 subpixels for Band 3. Distortions are different between Bands 1, 2, and 4 to those in Band 3 images because of the different spatial resolutions (1 $\mathrm{km}$ and $500 \mathrm{~m}$, respectively). Comparing the distortion patterns of each band, similarities among them were found. In short, the distortion of other bands can be estimated accurately from the result of Band 3 because the resolution of Band 3 images is higher than the others. This is apparent if the images in Fig. 4(g) and 4(h) are compared to those in Fig. 3(g) and 3(h), respectively.

The results of this study can contribute to image quality improvement and removal of clouds or other objects appearing over a short period of time. Additionally, because the image-to-image distortion of Band 3 is larger than that of other bands, it is possible to register temporal images of Band 1,2, and 4 precisely using the relation of Band 3 temporal images.

\section{Summary and Conclusions}

Besides the meteorological field, Himawari-8/AHI data is expected to be used in various other fields. The Earth's surface cannot always be observed freely by optical sensors due to cloud cover. However, Himawari-8 can observe the Earth's surface almost anytime, and with improved resolution, it can contribute to investigation and research on the urban environment, for example. The results of this study indicate that it is possible to extract more detailed images gathered by Himawari-8/AHI by applying multi-frame processing techniques.

\section{Acknowledgments}

Himawari- 8 data used in this study was provided by the NICT Science Cloud at the National Institute of Information and Communications Technology (NICT).

\section{References}

1) Himawari-8 is scheduled to start operation at 02 UTC on 7 July 2015, News Releases, Japan Meteorological Agency, http://www.jma.go.jp/jma/jma-eng/satellite/news/himawari89/2015 0707_himawari-8_operation_initiated.pdf (accessed September 7, 2017).

2) About quality improvement of Himawari-8 observation data, News Releases, Japan Meteorological Agency, http:/www.data.jma.go.jp/mscweb/en/operation8/eventlog/201603 09_himawari-8_event_en.pdf (accessed September 7, 2017).

3) Geostationary meteorological satellites -Himawari-8/9-, http://www.jma.go.jp/jma/kishou/books/himawari/201703_leaflet8 9.pdf, (accessed June 3, 2016).

4) Himawari-8/9 Data Users Guide, Japan Meteorological Agency, http://www.data.jma.go.jp/mscweb/en/himawari89/space_segment/ hsd_sample/HS_D_users_guide_en_v12.pdf (accessed September 7, 2017).

5) Nonaka, T., Matsunaga, T., Sawabe, Y., Moriyama, M., and Tonooka, H. : Radiometric Calibration of Thermal Infrared Bands of Terra ASTER and Landsat-7 ETM+ Using Hot Ground Targets, Journal of The Remote Sensing Society of Japan, 22 (2002), pp. 62-78 (in Japanese).

6) Sugimura, T., Uchida, Y., Aoyama, S., and Asaka, T. : Super Resolution of Himawari-8 2.5 minutes interval images, Proceedings of the 60th Spring Conference of the RSSJ, pp. 187-188, 2016 (in Japanese).

7) Sugimura, T., Uchida, Y., Aoyama, S., and Asaka, T. : Multiframe image processing using Himawari-8 2.5 minutes interval images, Proceedings of the 61st Autumn Conference of the RSSJ, pp. 187-188, 2016 (in Japanese).

8) Park, S. C., Park, M. K., and Kang, M. G. : Super-Resolution Image Reconstruction, IEEE Signal Processing Magazine, 20 (2003), pp. 21-36.

9) Irani, M., and Peleg, S. : Improving Resolution by Image Registration, CVGIP Graphical Models and Image Processing 53 (1991), pp. 231-239.

10) Misaizu, H., and Inamura, M. : Super-Resolution Reconstruction from the Incompletely Observed Remote Sensing Images, Journal of The Remote Sensing Society of Japan, 29 (2009), pp. 485-496 (in Japanese).

11) Carmen, M., and Inamura, M. : Improvement of Remotely Sensed Low Spatial Resolution Images by Back-propagated Neural Networks using Data Fusion Techniques, International Journal of Remote Sensing, 22 (2001), pp. 629-642.

12) Lu, Y., and Inamura, M. : Spatial resolution improvement of remote sensing images by fusion of subpixel-shifted multi-observation images, International Journal of Remote Sensing, 24 (2003), pp. 4647-4660.

13) Merino, M., and Nunez, J. : Super-resolution of remotely sensed images with variable-pixel linear reconstruction, IEEE Trans. Geoscience and Remote Sensing, 45 (2007), pp. 1446-1457.

14) Elad, M., and Feuer, A. : Restoration of a single super resolution image from several blurred, noisy and under sampled measured images, IEEE Trans. Image Processing, 6 (1997), pp.1646-1658. 